Projets

de paysage

\section{Projets de paysage}

Revue scientifique sur la conception et l'aménagement de l'espace

$18 \mid 2018$

Paysage et didactique

\title{
Une controverse d'aménagement urbain relue par le prisme d'une didactique du paysage
}

Le Grand Projet des Cherpines (agglomération de Genève, Suisse)

An urban development controversy reinterpreted through the prism of a didacticism of the landscape - The Cherpines major urban planning project (agglomeration of Geneva, Switzerland)

\section{Anne Sgard et Sylvie Paradis}

\section{OpenEdition}

\section{Journals}

Édition électronique

URL : http://journals.openedition.org/paysage/1023

DOI : 10.4000/paysage. 1023

ISSN : 1969-6124

Éditeur :

École nationale supérieure du paysage de Versailles-Marseille, Institut national des sciences appliquées Centre Val de Loire - École de la nature et du paysage, École nationale supérieure d'architecture et de paysage de Bordeaux, École nationale supérieure d'architecture et de paysage de Lille, Agrocampus Angers

\section{Référence électronique}

Anne Sgard et Sylvie Paradis, « Une controverse d'aménagement urbain relue par le prisme d'une didactique du paysage », Projets de paysage [En ligne], 18 | 2018, mis en ligne le 05 juillet 2018, consulté le 28 novembre 2019. URL : http://journals.openedition.org/paysage/1023 ; DOI : 10.4000/ paysage. 1023

Ce document a été généré automatiquement le 28 novembre 2019.

Projets de paysage 


\title{
Une controverse d'aménagement urbain relue par le prisme d'une didactique du paysage
}

\author{
Le Grand Projet des Cherpines (agglomération de Genève, Suisse) \\ An urban development controversy reinterpreted through the prism of a \\ didacticism of the landscape - The Cherpines major urban planning project \\ (agglomeration of Geneva, Switzerland)
}

Anne Sgard et Sylvie Paradis

1 Le projet de recherche "Didactique du paysage: mutualisation des expériences et perspectives didactiques à propos des controverses paysagères " réunit une dizaine d'enseignants-chercheurs en Suisse, en France, en Belgique et au Québec (Canada) ${ }^{1}$. Il vise à confronter des outils, des méthodes et des expériences de formation où le paysage est mobilisé, afin de mieux cerner ce qui pourrait alimenter une didactique commune du paysage, de concevoir de nouveaux outils et méthodes, d'améliorer les existants, et de proposer ces résultats aux milieux de la formation. Les membres de l'équipe de recherche sont tous formateurs, que ce soit à l'université (en géographie en particulier), en école du paysage, pour la formation continue ou en formation des enseignants du premier et du second degré. Ils font l'hypothèse que la confrontation des expériences autour d'un même objet peut nourrir une réflexion didactique commune et transversale.

2 Ce projet de recherche s'inscrit dans le contexte d'engouement pour les démarches participatives et de médiation, qui, de manière récurrente, en appellent à la sensibilisation, à la pédagogie et à l'éducation des habitants-citoyens. La Convention européenne $\mathrm{du}$ paysage (CEP, 2000) a du reste marqué un tournant en institutionnalisant cette nécessité participative (Jones, 2007). Sans intention de résumer ici une littérature abondante sur les démarches participatives, soulignons quelques retours d'expériences et de réflexions critiques sur les intérêts, potentiels et limites, de dispositifs conçus à propos de mesures ou de politiques paysagères ou 
utilisant le paysage comme outil de participation. Il s'agit en particulier d'Yves Michelin qui a depuis longtemps expérimenté différents dispositifs (voir notamment Michelin et Joliveau, 2005); Marie-Josée Fortin qui s'est penchée sur les conflits à propos des éoliennes et les enjeux de participation (Fortin et al., 2010); Laurent Lelli et Philippe Sahuc qui ont proposé les démarches itinérantes des "tréteaux du paysage " (2009) de même qu'Alexis Pernet et les «ateliers paysage » (2009); ou encore Hervé Davodeau qui s'est consacré à l'analyse des controverses et des démarches de médiation (Davodeau et Toublanc, 2010 ; Davodeau et Sant'Anna, 2011 ; Davodeau et Montembault, 2012). Tous relèvent à la fois les opportunités qu'offre le paysage en termes de mobilisation des acteurs, de facilitation du dialogue, de transversalité ; mais tous insistent également sur les ambiguïtés et dérives inhérentes à ces dispositifs. Nous ne reviendrons pas ici sur les démarches proprement dites mais sur ce que cet engouement et ce travail critique impliquent en termes de métiers et de formations.

Nous envisageons le champ de la médiation au sens large, telle que proposée par Yves Michelin (dans Candau et Michelin, 2009) : «La prise en compte de regards différents sur l'espace pour favoriser l'élaboration d'une action localisée ou d'un projet collectif. » Le projet de recherche "Didactique du paysage» entend ainsi observer différentes démarches selon une posture à la fois expérimentale et critique. Le contexte actuel interpelle les formateurs : à quelles connaissances et compétences renvoie ce rôle de " citoyen participatif »? En quoi ces injonctions à la participation modifient-elles en profondeur les métiers de l'aménagement, du développement territorial et du paysage? L'équipe s'appuie sur un objet transversal, la controverse publique, qui lui permet d'aborder le paysage d'emblée dans ses dimensions politiques, de s'intéresser aux systèmes d'acteurs, à la place de "l'expert » (paysagiste, géographe...), tout en questionnant le rôle du paysage dans ces controverses : peut-il contribuer à renouveler les modalités de gouvernance ? Comment les acteurs le mobilisent-ils ? À quoi leur sertil ? Cela nourrit les deux autres hypothèses du programme et de l'expérimentation présentée ici : la pertinence des situations de controverse où le paysage est mis en débat, explicitement ou non, et le caractère fondamentalement politique du paysage ainsi approché (Trom, 2001).

Dans ce cadre de recherche, un projet d'aménagement en marge de l'agglomération de Genève (Suisse) a été choisi : celui du Grand Projet des Cherpines. Cet exemple devait permettre aux chercheurs participants de confronter leurs regards et leurs méthodes et d'analyser une controverse selon une problématique décalée: analyser non pas comment elle émerge et se déploie, tel que pourrait le faire la sociologie des problèmes publics, mais en quoi elle peut être un objet pertinent de formation. Lors d'un workshop organisé à Genève du 9 au 11 novembre 2015, l'équipe de recherche a analysé ce cas d'étude à partir d'une mallette pédagogique, a arpenté le terrain et a proposé des séquences pédagogiques. L'objectif de cet exercice était de tester la pertinence de la controverse comme outil de formation et d'analyser la place, le rôle et les perspectives didactiques du paysage à partir de cet exemple de projet d'urbanisation. Cet article souhaite se pencher sur cette expérience en discutant les dimensions successives de cette mise en abîme, depuis la conception de l'exercice en amont, en vue de la mutualisation des regards des enseignants-chercheurs, jusqu'à l'analyse des propositions de dispositifs didactiques. Cette analyse nourrit un bilan réflexif sur ces productions : peut-on envisager une didactique commune ? À quoi sert le paysage dans ces dispositifs et au-delà dans les formations? 


\section{Le choix d'un terrain collectif où croiser les regards, expériences et intentions pédagogiques : un projet urbain, un projet dans les marges, un paysage ordinaire}

5 Une première étape de l'expérimentation consistait dans le choix d'un terrain approprié pour valider notre première hypothèse: la pertinence des situations de controverse pour approcher le paysage. La réflexion sur les controverses, ou plus largement sur les « questions socialement vives » (QSV), a été menée par les didactiques du monde social et de la nature (Albe, 2008, 2009; Legardez et Simmonneaux, 2003, 2011 ; Sgard dans Audigier et al., 2015). Ces QSV répondent avant tout à l'exigence d'ancrage de l'enseignement dans la société, dans l'actualité, dans des situations de débat. En outre, celles-ci permettent aux apprenants, qu'il s'agisse d'étudiants, de futurs paysagistes ou de scolaires, ou même de professionnels, d'entrer de plain-pied dans un questionnement sur les enjeux, les systèmes d'acteurs, les processus de décisions. Ce choix renvoie à un postulat de cette recherche: l'affirmation de la dimension fondamentalement politique du paysage car il questionne l'espace public (Besse, 2009; Rudaz et Sgard, 2016). Il s'agit donc d'identifier un terrain d'expérimentation didactique qui réponde à trois critères : que ce soit un objet de débat public, tout en bénéficiant de suffisamment de recul et d'informations pour pouvoir le donner à voir et à étudier (un projet à une étape pertinente du processus), et que le terrain pose des «questions paysagères », c'est-à-dire qu'il permette de problématiser par le prisme du paysage. C'est ce qui a présidé au choix du projet dit des Cherpines.

\section{Un projet emblématique pour Genève, un laboratoire de politique urbaine}

6 Le projet des Cherpines apparaît comme un cas "pilote » au sein de l'agglomération franco-valdo-genevoise (FGV), confrontée à des besoins urgents en logement à une échelle transfrontalière. En effet, ce futur "quartier durable» (DALE, 2014) se veut exemplaire, notamment en raison de sa taille ( 58 ha, 3000 logements et 2500 emplois à créer) et de ses exigences qualitatives en matière de quartier durable (qualité des constructions, mobilités douces, espaces verts...). Jusqu'à présent, les projets d'aménagement genevois étaient de tailles plus modestes, concernant une seule commune et impliquant des montages réglementaires plus légers. Les Cherpines représentent donc pour les acteurs locaux une expérimentation hors norme. En outre, ce projet articule plusieurs échelons institutionnels : les deux communes périurbaines sur lesquelles se situe le périmètre (Confignon et Plan-les-Ouates), la ville de Genève, le canton de Genève et l'agglomération transfrontalière dite du "Grand Genève ", auxquels s'ajoute ponctuellement la Confédération qui fixe des objectifs généraux (notamment du point de vue de la densification). 
Figure 1. Localisation du site des Cherpines, Grand Genève, Suisse

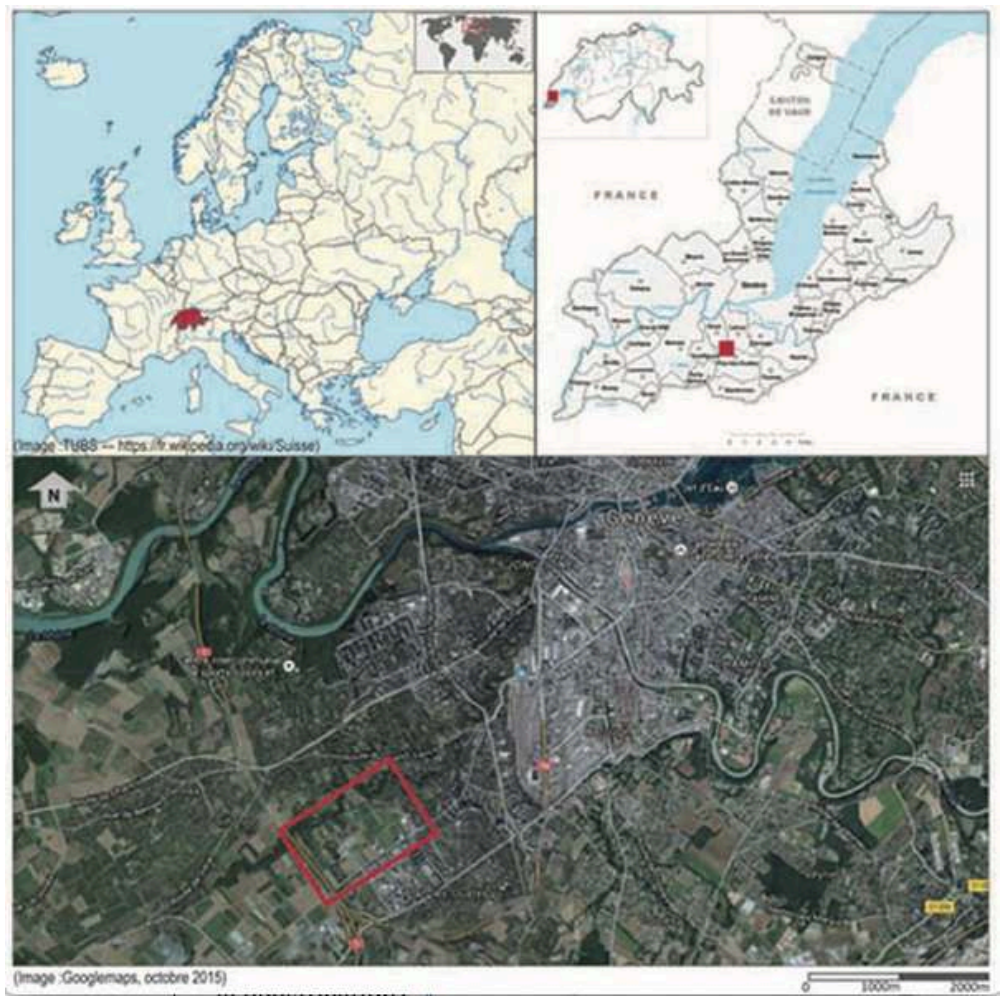

7 De plus, ce projet implique un déclassement de terres agricoles pour rendre le secteur urbanisable, ce qui n'est pas sans susciter le débat: le projet ne peut être " acceptable " que s'il permet une densification du futur quartier suffisamment importante pour compenser une perte de terres arables. En termes d'aménagement du territoire, si les besoins en logements sont une urgence dans le Grand Genève, ils sont mis en tension avec la préservation d'une agriculture permettant de nourrir la ville. À l'échelle genevoise et suisse, cette forme de sanctuarisation des terres agricoles n'est pas que technique et administrative, elle est fortement défendue par les habitants qui associent des qualités paysagères et un attachement symbolique à cette exigence d'autonomie alimentaire (Ernwein et Salomon Cavin, 2014).

8 L'obtention d'un déclassement des terres agricoles du site des Cherpines est donc une étape obligatoire. Dans un premier temps, en 2010, le Grand Conseil genevois décide ce déclassement, mais une coopérative sociale de maraîchers, présente sur le site, s'y oppose et initie la création d'un comité référendaire, réclamant une votation. Cela marque le point de départ de la controverse. Les différents acteurs mobilisés (associations, partis politiques...), pour ou contre, mènent alors une campagne active par le biais d'affiches, de blogs, ou d'articles dans la presse. 
Figure 2. Panneaux d'information de l'association Bienvenue aux Cherpines, et affiche d'opposition fixée sur un portail, sur place
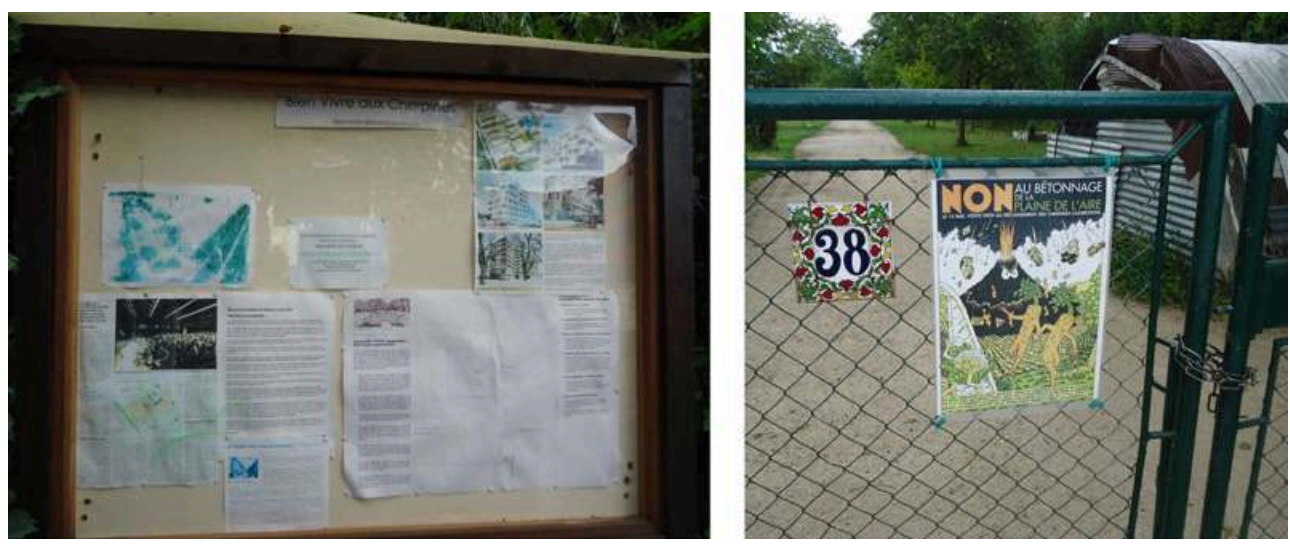

(C) Sylvie Paradis, août 2015

Le vote citoyen a lieu le 15 mai 2011, à l'échelle du canton. Il se solde par l'acceptation du déclassement à 56,6\%. Les communes les plus touchées par le projet des Cherpines y sont majoritairement opposées. Plus on s'éloigne, plus les communes sont favorables au déclassement. Depuis, le projet d'urbanisation a poursuivi son avancement, en franchissant différentes étapes : concours d'idées, plan d'aménagement assez précis à l'échelon de l'îlot, etc. Pour autant, rien n'est sorti de terre, les associations restent actives et la controverse ne semble pas terminée...

\section{Un projet saisi à une étape clé}

10 En 2015, le projet et la controverse sont à un point d'orgue. Quelques choix d'aménagement et lieux emblématiques se sont dégagés, susceptibles de focaliser les conflits ou les accords: une vaste place centrale, une desserte par une extension du tram, une grande diagonale devant relier les deux communes entre elles...

Si les associations de riverains ont pris acte de la décision de déclassement, elles tentent d'influer sur la conception du projet, pesant notamment sur les critères de quartier durable. Elles portent également un jugement amer sur la concertation qui s'est délitée dans le temps, s'estimant peu entendues.

Ce moment est propice à notre expérimentation dans la mesure où l'on peut retracer les étapes de ce projet, les acteurs et les argumentaires ; plusieurs chercheurs genevois se sont en outre penchés sur ce cas (Matthey et al., 2012 ; Gallezot, 2013) et en font un projet richement documenté. Une « histoire naturelle » (Callon, 1986) de la controverse peut être en grande partie retracée, même si elle n'est pas encore close. 


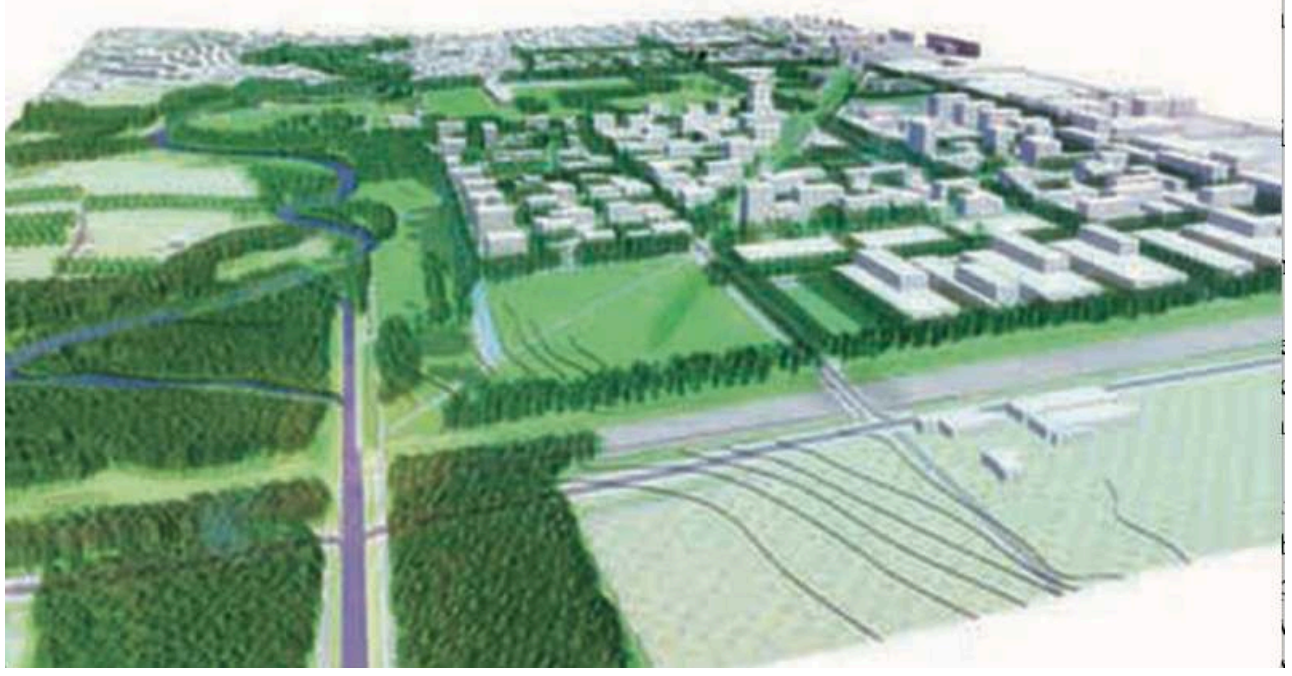

Sources : Extrait du plan directeur de quartier, cahier 2.03, 20 février 2013, p. 43.

\section{Un espace en attente, un paysage en devenir} découverte actuelle du terrain où rien n'est visible mais où tout est en attente est particulièrement stimulante pour notre recherche. Ce vaste périmètre se répartit entre parcelles en partie encore cultivées (pour beaucoup couvertes de serres, partiellement abandonnées), espaces de loisirs (terrains de football, centre équestre...) et petits groupes de villas de faible densité entourées de jardins privatifs.

Ce paysage est ainsi caractéristique des lisières urbaines (Bonin et al., 2016) en marge d'une ville toute proche; il est limité par des quartiers résidentiels et une vaste zone industrielle (côté Plan-les-Ouates), une autoroute et une rivière, l'Aire, qui a fait l'objet d'un projet de " renaturation ${ }^{2}$ ", marquent la transition avec la campagne voisine (côté Confignon) et la France. À l'échelle micro, quand on circule dans le périmètre, les impressions d'hétérogénéité, d'ambiances juxtaposées, de limites et de transitions dominent. C'était le choix initial de l'équipe de travailler sur un paysage ordinaire (Luginbühl, 1989 ; Sansot, 1989 ; Lelli et Paradis-Maindive, 2000) en périphérie urbaine : ce terrain y répond, tout en combinant ce paysage immédiat, banal, à portée de main, avec des perspectives lointaines sur le Salève au sud (montagne emblématique genevoise), voire sur le massif du Mont-Blanc par temps clair, et la crête du Jura au nord. 
Figure 4. Les lisières du site avant projet (2015)
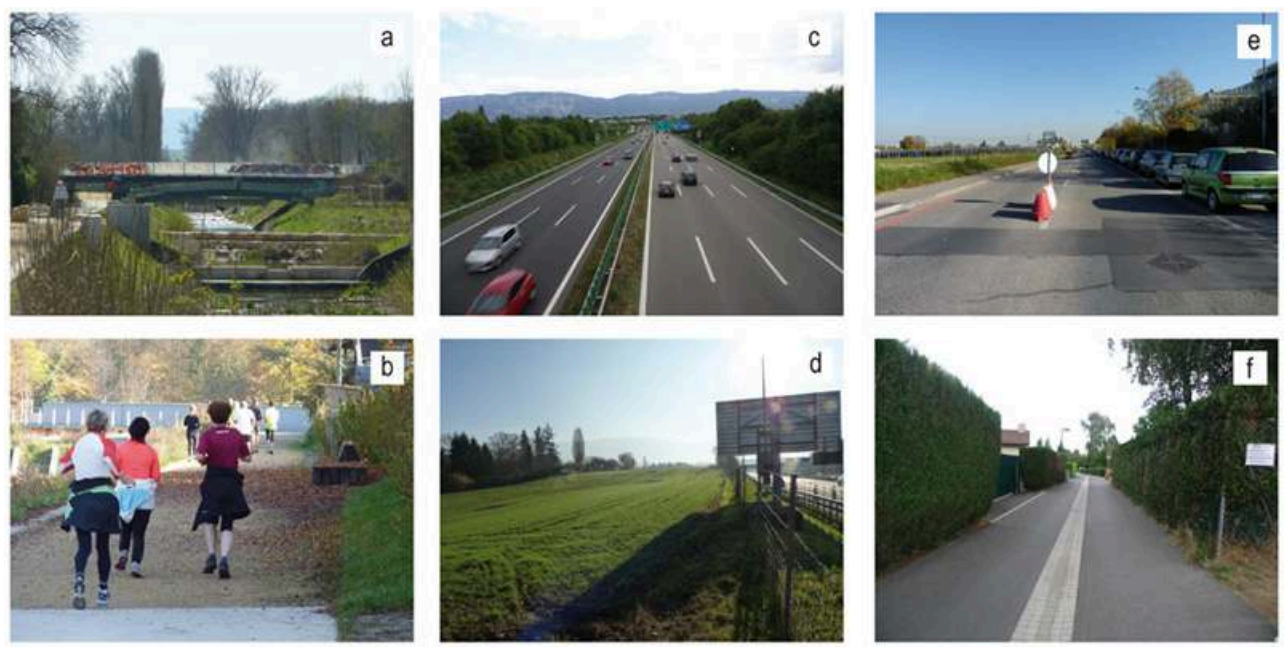

a. et b. L'Aire (rivière) suite à sa « renaturation ».

c. et d. L'autoroute A1.

e. La zone industrielle Plan-les-Ouates (ZIPLO) et la route de Base.

f. Le quartier de petites villas des Verjus.

Sources : Simon Gaberell, avril 2015 et Pierre Dérioz, novembre 2015 (a et b) ; Sylvie Paradis, août 2015 et Pierre Dérioz, novembre 2015 (c et d) ; Pierre Dérioz, novembre 2015 (e) ; Sylvie Paradis, août 2015 (f).

Vaste espace ouvert, entre une agriculture résiduelle et des équipements de loisirs, les Cherpines sont fréquentées assidûment par les riverains et par des Genevois qui y trouvent un espace de détente pour la famille, à moins de $5 \mathrm{~km}$ du centre de Genève.

Sur ce terrain, il s'agissait pour l'équipe organisatrice de concevoir un dispositif spatial double : un cas d'étude commun où soient mis en lumière les acteurs, enjeux, processus d'une politique urbaine, dans un espace bien identifié ; et en même temps un dispositif didactique qui mette à disposition des chercheurs-formateurs un objet de mutualisation, rapidement appréhendé, pour permettre la réflexion didactique.

\section{Comment transformer un laboratoire de politique urbaine en dispositif de formation?}

17 L'expérimentation s'est déroulée sur trois jours sous la forme d'un atelier de terrain. Une première demi-journée était consacrée à une présentation synthétique du contexte (grands principes d'aménagement suisses et genevois notamment), des différentes étapes du projet et de la controverse, afin que les participants acquièrent en accéléré une connaissance de cet exemple en vue de la réflexion didactique. Une seconde journée s'est déroulée in situ: découverte du terrain le matin, puis après-midi consacrée à la conception puis à la présentation d'activités pédagogiques, les publics visés étant laissés au choix. Le debriefing des propositions a eu lieu le troisième jour.

\section{Quelle préparation ? Quelle mallette pédagogique?}

18 L'enjeu consiste pour l'équipe organisatrice à sélectionner les documents clés, à propos du projet et de la controverse, nécessaires à la prise en main du terrain par les chercheurs en un temps réduit (voir encart 1). Au-delà du choix en termes de nombre, 
d'accessibilité, de précision des documents, cette étape implique une réflexion sur le contact avec le terrain, question-clé pour le géographe ou le paysagiste. Comment pondérer le niveau de connaissances factuelles en amont et la nécessaire découverte in situ ? Comment aider à l'appréhension du cas sans « déflorer » ou même «noyer »? Le géographe et le paysagiste ont en commun ce rapport à la fois intime, sensoriel et expert avec le terrain, cœur de leur pratique professionnelle. Ici, ils doivent à la fois expérimenter la découverte d'un terrain nouveau (et d'un contexte de projet différent pour la plupart d'entre eux car non suisses) et l'appréhender en tant que terrain potentiel de formation, ce qui implique d'associer des compétences mises en œuvre (percevoir, interpréter, organiser, exprimer, problématiser...) et une posture de transmission : comment former à ces compétences?

Encart 1. Présentation de la mallette pédagogique de l'exercice (novembre 2015)

Le choix du corpus de documents proposés dans la mallette distribuée
aux participants du workshop s'est porté sur un assemblage incluant
des documents permettant une compréhension du projet d'une part,
et des documents potentiellement utilisables dans le cadre des
propositions d'activités pédagogiques d'autres part. Selon nos critères,
il fallait que ces éléments soient en nombre limité, facilement
appréhendables et inspirants (comme supports éventuels d'activités
pédagogiques).
II s'agissait :
De trois photos aériennes localisant le site à différentes échelles
(agglomération, communes, quartier)
D'une synthèse (reprise du diaporama préalable présenté aux
participants) présentant le contexte d'aménagement suisse et
genevois, les caractéristiques du site avant-projet (limites, etc.) et
les principales étapes et caractéristiques du projet d'urbanisation
De tableaux de synthèse thématique issus des entretiens semi-
directifs menés en préalable par l'équipe organisatrice, posant les
" pour » et les « contre » de la controverse avec quelques extraits
courts de dires d'acteurs. Ce format a été préféré à une série
d'extraits d'entretiens plus longs, juxtaposés, qui n'auraient pas pu
illustrer de façon suffisamment complète et succincte la diversité des
positions dans le temps.
D'une sélection d'affiches et illustrations produites lors du référendum,
représentant les pour et contre de la controverse
De deux articles scientifiques (Matthey et al. 2012 ; Salomon Cavin
2012 ) présentant le contexte du projet, et des éléments d'analyse
de la controverse.

\section{De la découverte du terrain à la transposition didactique}

19 La découverte du terrain par les participants s'est faite en autonomie, chacun muni de sa mallette pédagogique, choisissant le rythme et l'itinéraire. Cela a été préféré à une visite commentée, pour susciter la réflexion sur les compétences attendues et les apprentissages à développer à partir du cas d'étude. Chacun devait alors parcourir le site tout en réfléchissant à une proposition pédagogique. Aucune rencontre d'acteurs n'a été prévue, les entretiens ayant été faits au préalable par l'équipe organisatrice et présentés de manière synthétique ${ }^{3}$.

Une impression commune s'est dégagée de la découverte du terrain : le décalage entre le ressenti suite à l'arpentage et les représentations du projet vues précédemment, cartes, plans et dessins. Le projet d'urbanisation des Cherpines transforme 
profondément le site et ne conserve que très peu les caractères actuels : les sensations d'un vaste espace ouvert mais pas "vide" pour autant, l'agriculture résiduelle mais visuellement très présente, avec sa trame parcellaire et ses chemins, et surtout les spécificités d'un paysage de marge urbaine, une hétérogénéité d'ambiances, une mosaïque de paysages avec des cheminements variés, des transitions, des échappées... Les participants y ont perçu un paysage en suspens, avec des traces d'abandon, des signes de changement, une situation d'attente, des espaces d'entre-deux entre ville et campagne. Tous ont été frappés par la fréquentation de ce site, par la diversité des pratiques (promenade, jogging, vélo, équitation, foot...).

Dans leurs propositions pédagogiques, les enseignants-chercheurs ont tous fait le choix de mettre l'accent sur ce caractère des lieux, pour amener l'apprenant à le percevoir, à réagir sur ses perceptions, et à confronter ce ressenti aux projets. Leurs propositions convergent presque toutes sur une approche sensible et sensorielle et une mise en débat du paysage.

Figure 5. Ambiances et composants du site avant projet (2015)
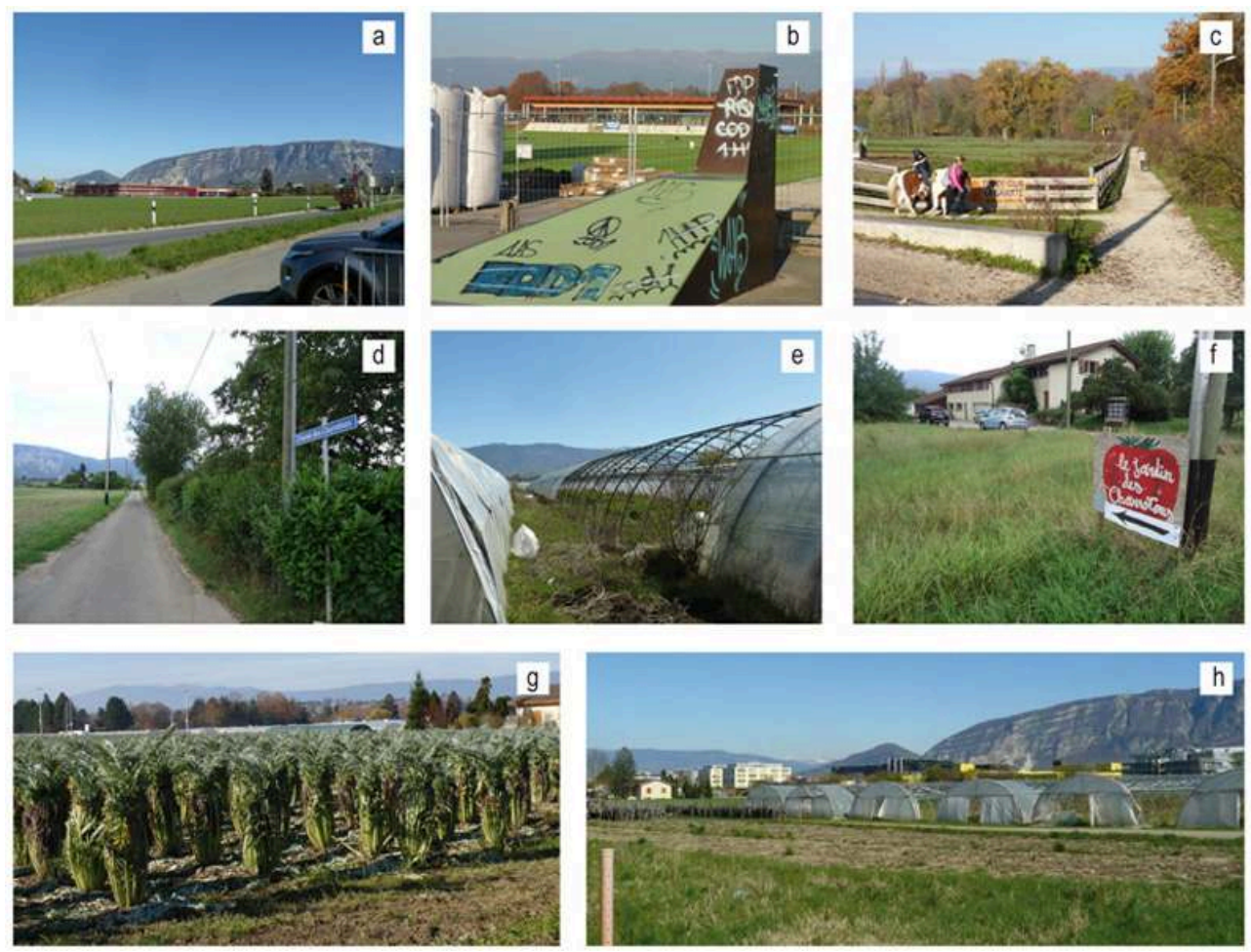

a. L'école Aimée Stitelmann avec le Salève en fond.

b. Le centre sportif des Cherpines.

c. Le centre équestre de la Gavotte.

d. Chemin des Charrotons, fragments d'une agriculture résiduelle plus ou moins dynamique.

e. Des serres à l'abandon.

f. La coopérative des Charrotons.

g. Culture labellisée du cardon argenté

h. Maraîchage près du centre sportif et vue sur le Salève.

Sources : Simon Gaberell, avril 2015 (a et e) ; Pierre Dérioz, novembre 2015 (b, c et g) ; Sylvie Paradis, août 2015 (d et f) ; Anne Sgard, août 2015 (h) 


\section{L'approche sensible et la mise en débat} didactique aujourd'hui bien connu et expérimenté, autant en contexte scolaire que dans les formations professionnelles et en aménagement. Dans les propositions élaborées, il prend des formes diverses mais vise toujours à expérimenter des démarches participatives, par exemple :

- « concevoir une réunion publique » à propos des qualités du site : quelles sont les qualités du lieu pour les acteurs en présence ? Il s'agit alors de déterminer les acteurs et d'établir l'ordre du jour ;

- «faire travailler deux équipes sur le même site » : une équipe « diagnostic » qui propose une restitution à une équipe « acteurs » qui doit réagir ;

- « organiser une confrontation de scénario » : chaque groupe conçoit un projet sous la forme d'une maquette et le défend...

Ici, le jeu de rôles doit permettre d'abord la mise en débat: des perceptions des lieux, des pratiques et modalités d'appropriation, des choix d'aménagement... Il amène la verbalisation des impressions, la recherche de la posture et du vocabulaire adéquats, l'argumentation, la recherche de consensus ou l'exploration des désaccords... Il ouvre également à une réflexion sur le positionnement de «l'expert » et sur les outils de l'expertise. Il permet par la répartition des rôles une certaine prise de conscience des jeux d'acteurs, de la place des professionnels (élus, techniciens, conseillers, médiateurs...), dans des situations de médiation paysagère. Avec des étudiants avancés, il peut intégrer un moment de debriefing : revenir de manière critique sur les attendus, les formes et modalités, le déroulement, les limites et dérives de ces dispositifs. Et permettre ainsi une institutionnalisation ${ }^{4}$ : « qu'avons-nous appris?».

Projets de paysage, 18 | 2018 


\section{Retour réflexif : à quoi sert le paysage?} ans les propos des acteurs, il s'exprime autrement : à travers un discours sur le cadre de vie quotidien, sur des pratiques inscrites dans un vaste espace de "nature » et de «liberté » rare à Genève, sur des formes d'appropriation, parfois des souvenirs, une mémoire familiale... Ce cas donne à voir un rapport au lieu, affectif et sensible. En outre, cet exemple permet de travailler sur des types de paysage intéressants du fait de leur discrétion, de leur banalité et de leur apparent effacement dans les enjeux : quels ressentis, perceptions et représentations cohabitent ou se heurtent? Au-delà de l'esthétique, en quoi le paysage est-il un « espace social habité » (Paquette et al., 2005) ?

31 Les activités peuvent donc s'appuyer sur ce cas pour faire réfléchir les apprenants sur le paysage ordinaire : quelle place peut-il avoir, d'une part dans la mobilisation contre un projet ou des aspects précis du projet, d'autre part dans la concertation à mettre en place ? Par exemple, pour les élèves de niveau secondaire, voire primaire, habitants du quartier, ce qui pourrait apparaître comme un obstacle devient au contraire un atout: 
c'est un paysage familier, immédiatement compréhensible et décrypté. Les enjeux liés au projet de nouveau quartier sont facilement saisis. L'intérêt de travailler sur une controverse de proximité est de mettre en œuvre une réflexion sur les acteurs : qui s'exprime? Quelles alliances/oppositions apparaissent? La finalité est qu'ils comprennent que l'espace urbain est le produit d'une histoire sur le temps long mais aussi de politiques d'aménagement, de décisions, de choix. Cela vient à l'encontre de représentations de la ville que peuvent avoir les élèves, tantôt attractives, tantôt effrayantes, toujours difficiles à saisir.

Ces constats expliquent le choix opéré par la plupart des membres de l'équipe d'une approche par les sens. L'enjeu est ainsi d'amener les apprenants à faire le lien entre le sensible, l'esthétique ordinaire, le sentiment d'attachement et le politique, à considérer le droit des usagers à défendre un certain rapport au territoire, même s'il n'y a pas de valeurs patrimoniales ou remarquables à défendre. La controverse vise à faire prendre conscience que la mobilisation des habitants peut intervenir dans des sites a priori sans valeur affirmée et acquérir une dimension médiatique inattendue. Le passage par des jeux de rôles permet de tester in vivo ces argumentaires dans une simulation de débat.

\section{Comment et pourquoi former à un paysage conçu comme outil politique?}

En arrière-plan de cette recherche, on l'a dit, se trouve la préoccupation de l'équipe pour la formation (de paysagistes, de géographes, d'aménagistes) à des démarches de médiation, estimant que la multiplication de ces démarches implique une transformation profonde de ces métiers. Pierre Donadieu estime que « le paysagisme de médiation met en place des dispositifs sociopolitiques qui permettent d'élaborer des actions publiques régulatrices de la production des paysages et des lieux. Il renonce à élaborer des projets sans le contexte des intentions politiques qui leur donnent un sens et contribuent en principe à les renouveler " (2009). Hervé Davodeau (dans Rudaz et Sgard, 2016) rappelle que les élèves paysagistes doivent "faire avec » la dimension politique alors que dans leur formation, la pédagogie tend plutôt au contraire à "survaloriser les capacités d'imagination et d'inventivité de l'étudiant» et que «l'enseignement du projet est d'abord un espace de liberté et d'accomplissement personnel ». Quel que soit le métier, une posture de médiation implique un retrait du concepteur ou de l'expert, au profit d'un rôle de porte-parole ou porte-crayon pour coconstruire la réponse, pour favoriser le partage (Candau et Michelin, 2009). Les futurs "médiateurs » ont dès lors besoin d'adopter une lecture politique du paysage et d'en acquérir les outils: prendre la mesure des enjeux idéologiques, symboliques et potentiellement conflictuels du projet, identifier les systèmes d'acteurs, les jeux de pouvoir et les formes d'instrumentalisation.

Ces situations sont largement dépendantes $\mathrm{du}$ contexte politique, légal et réglementaire des interventions. La Suisse offre un cadre intéressant car elle combine une tradition de la consultation populaire et un attachement très fortement exprimé de la nation à ses paysages. Cela pourrait amener à penser que les démarches de médiation y sont moins nécessaires car les votations permettent l'intervention efficace des citoyens dans les processus décisionnels. Le cas des Cherpines montre qu'il n'en est rien. Les situations de blocage sont fréquentes et souvent longues, du moins dans le cas du canton de Genève ${ }^{5}$, et rares sont les projets d'aménagement qui y échappent. Outre 
les votations communales ou cantonales qui concernent fréquemment ce type de projets, les étapes finales des projets (les plans localisés de quartier - PLQ notamment) peuvent donner lieu à des procédures de recours, qui sont elles aussi fréquentes, et peuvent faire avorter un projet malgré son avancement et les investissements importants en amont (humains, financiers, techniques). Les professionnels de l'aménagement en appellent donc de plus en plus à des démarches de médiation pour anticiper ces blocages, intégrer mieux en amont les populations concernées (Dewarrat et al., 2003). De la même manière, si le récit national suisse a largement utilisé les emblèmes paysagers des montagnes alpines, ce discours privilégie les paysages naturels ou ruraux et les paysages remarquables patrimonialisés (Walter, 1991). La notion de paysage ordinaire reste peu répandue encore (Sgard dans Rudaz et Sgard, 2016) et l'idée que le paysage puisse être une catégorie de l'action publique doit encore faire son chemin (Ruegg et Letissier dans Rudaz et Sgard, 2016), ce malgré la récente entrée en vigueur de la CEP en Suisse ( $1^{\mathrm{er}}$ juin 2013).

Revenons aux métiers et à la formation. Cette posture de coconstruction du projet implique aussi d'identifier les savoirs mis en œuvre dans ces situations et, en retour, les compétences attendues de la part des professionnels du paysage et/ou de l'aménagement. Si la réponse à la commande revendique une coconstruction, entre experts, décideurs et usagers, pour utiliser des catégories sommaires, elle fait appel à des "dispositifs sociopolitiques" (Donadieu, 2009) où sont confrontés une lecture du territoire et de ses usages, des données techniques (contexte budgétaire, cadre légal et réglementaire, contraintes économiques ou environnementales...), des choix et des arbitrages. Cette confrontation « met en système » des savoirs de natures diverses, car ceux-ci entrent en interaction, se nourrissent ou s'opposent les uns aux autres, en tout cas se transforment mutuellement et cela implique une dynamique dans la construction du projet. Les savoirs circulent et s'hybrident dans un processus de médiation à construire, où l'iconographie du paysage sert souvent d'intermédiaire à l'expression, à l'émulation et à l'élicitation (Paradis et Lelli, 2010). Plutôt que d'utiliser le couple savoirs profanes/savoirs savants, qui reste réducteur et repose sur une dichotomie qui n'évite pas une hiérarchie implicite, insistons surtout sur la diversité des formes de connaissances et de savoirs qui peuvent interagir: informations et connaissances, savoirs d'expériences et savoirs d'autorité, imagination et références, valeurs et faits, produits des émotions et de la distanciation, mémoire et anticipation... On le voit, former à cette posture ne peut ni se résumer en une somme de savoirs et savoir-faire techniques, ni donner libre cours à l'imagination créatrice : cela signifie apprendre à comprendre ce système et à y naviguer. L'apprenant mobilise de manière sans cesse revisitée des savoirs et des compétences professionnels, son imagination et sa capacité à susciter, à reconnaître (dans tous les sens du terme) et à interpréter la diversité des savoirs en interaction. Les finalités sont autant techniques que politiques et éthiques.

\section{Une didactique commune?}

\section{Diversité des publics et des démarches...}

Les publics ciblés par cette expérimentation des Cherpines sont divers et impliquent des objectifs adaptés. Si les différents publics arrivent avec des profils et des bagages variés, on constate que les compétences visées par les propositions de séquences 
conçues lors du workshop sont proches : il s'agit d'expérimenter le paysage comme un outil de gouvernance pertinent non pas malgré mais grâce à son ancrage dans le sensible, la subjectivité, les pratiques. Observer des pratiques, permettre le dialogue, confronter des ressentis, interpréter des sensibilités et les faire partager... L'objectif est de construire un système de savoirs, entre compréhension du lieu et des modes d'appropriation et compétences techniques, de l'objectiver pour y naviguer, c'est-à-dire pour intégrer et transcrire ces liens des habitants et usagers au territoire dans le projet. S'appuyer sur la plasticité du concept de paysage pour en explorer le potentiel de mise en système.

Pour les scolaires, bien que cela concerne peu de propositions, l'objectif est de renouveler en profondeur la place du paysage dans l'enseignement, d'autant qu'en Suisse le paysage n'apparaît presque plus dans le plan d'études romand (PER) ${ }^{6}$. Cette absence est délibérée de la part des concepteurs du PER : il s'agit de rompre avec une approche traditionnelle descriptive, naturaliste, non problématisée de l'espace où la lecture de paysage figurait en bonne place. Réintroduire le paysage signifie donc l'aborder non comme un cadre à décrire, mais comme un lien au territoire vécu par l'élève, dans lequel il peut se projeter pour comprendre (Janzi et Sgard, 2013). Les ressorts du sensible, des émotions (en collaboration avec les arts plastiques, avec le français, etc.) contribuent aux apprentissages. C'est en cela que l'entrée par la controverse trouve tout son intérêt : l'objet de la transposition est moins le paysage que l'aménagement de l'espace, les acteurs, les stratégies, les modalités. Le paysage devient outil et non objet d'apprentissage.

\section{... mais convergence des finalités}

Les finalités communes se dessinent, et concernent à la fois la conception du paysage et la question de la gouvernance. En effet, cet exemple des Cherpines a contribué à montrer l'intérêt d'une pédagogie mettant en scène le paysage ordinaire. Nombre d'étudiants (mais aussi les habitants si l'on se projette dans les démarches de médiation elles-mêmes), quel que soit leur âge, abordent spontanément le paysage comme un décor, volontiers naturel ou rural, à prendre en compte en fonction de valeurs patrimoniales, économiques ou en lien avec la biodiversité. En Suisse, les conséquences en sont à la fois consensuelles et convenues: protéger. Ce paysage sert donc difficilement une pédagogie fondée sur l'étonnement, le questionnement, la mise en tension, la problématisation (Fabre, 2011). Cet exemple permet en outre d'insister sur un paysage en mouvement, évolutif, et de se projeter dans l'avenir. Il ne s'agit pas de savoir quel paysage on veut conserver, mais quel paysage on veut collectivement pour demain.

La seconde finalité commune renvoie au cadre englobant de la gouvernance et à son analyse critique. L'insertion du paysage dans les politiques publiques a été relancée par la diffusion des politiques de développement durable et l'injonction aux démarches participatives ; la CEP est un jalon significatif. Dans le domaine scolaire, l'éducation au développement durable, injectée dans l'ensemble des systèmes scolaires depuis une dizaine d'années, vise la formation d'un citoyen informé, responsable et critique, apte au dialogue et à la participation. L'analyse de la controverse cherche ici à questionner le processus de décision: qui est légitime à décider? À quelle échelle se fait la participation? À quelle étape du processus ? Pour quelle prise en compte des avis des 
habitants/usagers? Qu'est-ce que la démocratie locale peut gagner de ces procédures ? La finalité citoyenne est donc explicite: il s'agit de former ceux qui seront aptes à concevoir et à critiquer ces dispositifs et ceux qui, on l'espère, s'y exprimeront.

Enfin ne négligeons pas les finalités plus méthodologiques : réfléchir avec les étudiants à l'expression du paysage, condition de sa mise en débat. Comment mettre des mots sur des formes, des ressentis, des émotions et les discuter? Comment parler et faire parler du paysage ? Quels autres supports utiliser quand les mots sont difficiles à trouver? Les dispositifs proposés leur permettent d'expérimenter eux-mêmes ces difficultés, pour imaginer d'autres démarches, notamment grâce à des expressions iconiques (dessin, carte sensible, photo, maquette...).

\section{Éléments de conclusion}

Une première conclusion se dégage. Le paysage est depuis longtemps objet et enjeu de politiques publiques; il s'impose de plus en plus comme outil de gouvernance des territoires: outil de diagnostic, outil de patrimonialisation, outil de marketing territorial... et plus récemment outil de négociation et de médiation. Cet élargissement de son champ peut être facteur de dilution autant que de renforcement de la thématique paysagère dans les projets de territoire. Il nous semble que la nécessité de réfléchir à la formation des professionnels (paysagistes, géographes, urbanistes, écologues, aménageurs...) qui œuvrent à sa mise en œuvre s'impose aujourd'hui.

L'expérimentation présentée ici, peu courante, s'est déroulée sur un laps de temps très court, avec les contraintes inhérentes à ce type d'atelier, mais nous semble néanmoins concluante. Cette première étape a permis de stabiliser la méthode (choix du terrain, de la mallette, des dispositifs...), ce qui est déjà en soi un apport au programme ; il reste à valider ces apports en termes de transférabilité à d'autres cas d'étude.

L'objectif de mutualisation des expériences et de retour réflexif sur ces démarches est confirmé et doit être poursuivi : tous les formateurs témoignent de leurs habitudes, pratiques, tentatives, envies en termes de dispositifs ou de démarches pédagogiques mais tous relèvent la minceur de la bibliographie à ce sujet. Bien des aspects restent à l'état de routines plus ou moins implicites, ou de pratiques récurrentes mais non formalisées. Il manque des échanges sur les pratiques, des bilans critiques des résultats et de leur transfert en formation, une analyse fouillée des démarches d'évaluation... La didactique vise avant tout cette posture réflexive : prendre du recul, déconstruire ses démarches pour mieux les reconstruire, ouvrir et inventorier sa boîte à outils, formaliser et hiérarchiser ses objectifs, expliciter ses références et ses finalités. Ainsi cet atelier aux Cherpines met en lumière le rapport premier au terrain, les interactions entre perceptions et apprentissages, la question du langage et l'intérêt des jeux de rôles, la place de la transcription cartographique, plus globalement la pédagogie de l'atelier, et invite à y réfléchir collectivement.

Ainsi, au-delà de cet objectif de retour sur expériences, des questionnements plus proprement didactiques se dégagent et semblent prometteurs : analyser les situations de médiation en termes de circulation-transformation des savoirs, les dispositifs de problématisation et de mise en débat de la construction du problème, les modes d'institutionnalisation adaptés à une expérience de terrain et de médiation, les modalités et les critères d'évaluation des dispositifs de formations à la médiation. Nous 
ouvrons là un chantier, vaste et stimulant, auquel nous espérons convier chercheurs, praticiens et formateurs.

Nous tenons à remercier les enseignants-chercheurs qui ont participé au workshop des 9-11 novembre 2015 à Genève et sans qui cet article n'aurait pas été possible : Sophie Bonin (ENSP-LAREP Versailles, France), Laurent Daune (HEPIA Genève, Suisse), Hervé Davodeau (AgroCampus Ouest-ESO Angers, France), Pierre Dérioz (université d'Avignon CIRAD, France), Monique Toublanc (ENSP-LAREP Versailles, France), ainsi que Sandrine Billeau-Beuze (adjointe scientifique, université de Genève), François Diverneresse (doctorant, université de Genève, Suisse) et Simon Gaberell (aujourd'hui à HES-SO Genève, collaborateur scientifique de février à juillet 2015, université de Genève). Nous remercions également le Fonds national suisse de la recherche qui finance le programme de recherche à l'origine de ce texte (subside 10001A_156116/1, février 2015-janvier 2018, dirigé par Anne Sgard).

\section{BIBLIOGRAPHIE}

Albe, V., « Pour une éducation aux sciences citoyennes : une analyse sociale et épistémologique des controverses sur les changements climatiques », Aster, $n^{\circ} 46,2008$, p. 45-70.

Albe, V., Enseigner les controverses, Rennes, Presses universitaires de Rennes, 2009.

Audigier, F., Sgard, A., Tutiaux-Guillon, N. (dir.), Sciences de la nature et sciences de la société dans une école en mutation. Fragmentations, recompositions, nouvelles alliances?, Louvain-la-Neuve, De Boeck-Supérieur, 2015.

Besse, J.-M., Le Goût du monde. Exercices de paysage, Arles/Versailles, Actes Sud/ENSP, 2009.

Bonin, S., Toublanc, M., Dérioz, P., Béringuier, Ph., « Des franges du projet urbain au projet de frange urbaine », Projets de paysage, $n^{\circ} 13$, janvier 2016, URL : http://www.projetsdepaysage.fr/fr/ des_franges_du_projet_urbain_au_projet_de_frange_urbaine.

Callon, M., « Éléments pour une sociologie de la traduction. La domestication des coquilles SaintJacques et des marins-pêcheurs dans la baie de Saint-Brieuc ", Année Sociologique, n 36, 1986, p. 169-208.

Candau, J., Michelin, Y., « Paysage, outil de médiation », IFV (Institut français de la vigne et du vin), coll. « APPORT Agriculture et Paysage », n 8, décembre 2009.

Conseil de l'Europe, Convention européenne du paysage, Florence, 2000.

DALE, « Grand Projet des Cherpines », République et Canton de Genève/Commune de Confignon/ Commune de Plan-les-Ouates, mars 2014, URL : Http://ge.ch/amenagement/media/ amenagement/files/fichiers/images/documents/cherpines_4pages_final_web.pdf.

Davodeau, H., « Paysages et médiations, retour d'expériences pédagogiques », dans Guillot, X. (dir.), Espace rural et projet spatial, Saint-Étienne, Publications de l'université de Saint-Étienne, 2012, p. 161-166. 
Davodeau, H., Montembault, D., « La participation, un facteur de renouvellement des pratiques paysagistes? ", Actes du colloque Paysage en partage, Fondation Brayard Architectes, Genève, 25 avril 2012, p. 93-97.

Davodeau, H., Toublanc, M., « Le paysage outil, les outils du paysage, principes et méthodes de la médiation paysagère ", OPDE outils pour décider ensemble, aide à la décision et gouvernance, recueil des communications du colloque des 25-26 octobre à Montpellier, 2010, p. 375-391.

Davodeau, H., Sant'Anna, C., « La participation du public et ses incidences sur l'évolution des théories et pratiques du projet des paysagistes ", Territoires en mouvement, $\mathrm{n}^{\circ} 11,2011$, p. 50-65.

Dewarrat, J.-P., Quincerot, R., Weil, M., Woeffray, B., Paysages ordinaires. De la protection au projet, Sprimont, Mardaga, 2003.

Donadieu, P., «Où vont les architectes paysagistes? Du paysagisme jardiniste au paysagisme de médiation », Planum. The European Journal of Planning on-line, actes du colloque de Bari, 2009, URL : www.planum.net.

Droz, Y., Mieville-Ott, V., La Polyphonie du paysage, Lausanne, Presses polytechniques et universitaires romandes, 2005.

Ernwein, M., Salomon Cavin, J., « Au-delà de l'agrarisation de la ville : l'agriculture peut-elle être un outil d'aménagement urbain ? Discussion à partir de l'exemple genevois », Géocarrefour, 89, 1-2, 2014, URL : http://geocarrefour.revues.org/9380.

Fabre, M., Éduquer pour un monde problématique. La carte et la boussole, Paris, PUF, Coll. «L'interrogation philosophique », 2011.

Fortin, M.-J., Devanne, A.-S., Le Floch, S., « Le paysage politique pour territorialiser l'action publique et les projets de développement : le cas de l'éolien au Québec », Développement durable et territoires, vol. $1, \mathrm{n}^{\circ} 2,2010$.

Gallezot, H., « Zone 5 : une controverse en paysage », Projets de paysage, ${ }^{\circ}{ }^{9}$, décembre 2013, URL : http://www.projetsdepaysage.fr/fr/zone_5_une_controverse_en_paysage.

Grand Genève, « Projet d'agglo franco-valdo-genevois. Projet paysage 2 », Cahier $n^{\circ} 13-8$, annexe II, juin 2012.

Janzi, H., Sgard, A., «Le "savoir des questions" : comment problématiser avec les élèves ? Un exemple d'élément déclencheur : des éoliennes dans le paysage genevois », Penser l'éducation, hors-série décembre 2013, p. 205-221.

Jones, M., "The European Landscape Convention and the question of public participation ", Landscape Research, vol. 32, $\mathrm{n}^{\circ}$ 5, 2007, p. 613-633.

Legardez, A., Simmonneaux, L. (coord.), Développement durable et questions d'actualité. Questions socialement vives dans l'enseignement et la formation, Dijon, Éducagri éditions, 2011.

Legardez, A., Simmonneaux, L. (dir.), L'École à l'épreuve de l'actualité. Enseigner les questions vives, Paris, ESF, 2003, p. 19-33.

Lelli, L., Sahuc, P., « Quelle place pour les chercheurs dans un dispositif d'animation locale? L'exemple des tréteaux du paysage du Parc naturel régional des Causses du Quercy ", Revue d'Auvergne, vol. 123, n 590-591, 2009, p. 399-418.

Lelli, L., Paradis-Maindive, S., « Quand le paysage ordinaire devient un paysage remarqué », SudOuest européen, $n^{\circ} 7,2000$, p. 27-34. 
Luginbühl, Y., « Paysage élitaire et paysages ordinaires », Ethnologie française, n 3, 1989,

p. 227-238.

Matthey, L., Mager, C., Gaillard, D., « Chronique d'une controverse annoncée : le récit d'urbanisme à l'heure du développement urbain durable », Programme interdisciplinaire de recherches ville et environnement (PUCA, MEEDDAT), Genève, 2012.

Michelin, Y., Joliveau, T., « Le paysage au service de démarches participatives et prospectives de développement local : enseignements d'expériences de recherche-action conduites dans le Massif central », Revue d'Auvergne, n 571, 2005, p. 233-262.

Paquette, S., Poullaouec-Gonidec, P., Domon, G., « Le paysage, une qualification socioculturelle du territoire ", Revue d'histoire de la culture matérielle, nº 62, 2005, p. 60-72.

Paradis, S., Lelli, L., «La médiation paysagère, levier d'un développement territorial durable ? ", Développement durable et territoires, vol. 1, n 2, 2010, URL : http://

developpementdurable.revues.org/8548.

Pernet, A., « Une médiation paysagiste comme support de recherche : l'expérience de l'atelier des paysages en vallée de l'Ance, Parc naturel régional Livradois-Forez, Auvergne », Projets de paysage, $\mathrm{n}^{\circ}$ 3, décembre 2009, URL : http://www.projetsdepaysage.fr/

une_mediation_paysagiste_comme_support_de_recherche_1_experience_de_1_atelier_des_paysages_en_vallee_de_1_ance.

Rudaz, G., Sgard, A. (dir.), « Les dimensions politiques du paysage », Géo-regards, dossier n 8, Neuchâtel, 2016.

Salomon Cavin, J., « Entre ville stérile et ville fertile, l'émergence de l'agriculture urbaine en Suisse », Environnement urbain/Urban environment, vol. 6, 2012, URL : http://eue.revues.org/453.

Sansot, P., « Pour une esthétique des paysages ordinaires », Ethnologie française, $n^{\circ} 3,1989$, p. $239-243$.

Trom, D., « À l'épreuve du paysage. Constructivisme savant et sens commun constructiviste », Revue du Mauss, vol. 1, n 17, 2001, p. 247-260.

Walter, F., «La montagne des Suisses. Invention et usage d'une représentation paysagère (XVIII ${ }^{\mathrm{e}}$ XX ${ }^{\mathrm{e}}$ siècle) », Études rurales, n 121-124, 1991, p. 91-107.

\section{NOTES}

1. Voir le site web du projet : http://www.unige.ch/portail-didactique-paysage/.

2. Politique environnementale phare du canton.

3. Au total, 11 entretiens semi-directifs ont été réalisés par nos soins : 4 personnes des 2 communes (élus, services techniques), 2 personnes des services de l'État et canton de Genève, 2 entretiens auprès d'associations de riverains, 2 auprès d'acteurs agricoles, 1 bureau d'études ayant participé au processus de conception du projet.

4. Terme de didactique désignant le processus par lequel l'enseignant amène les élèves euxmêmes à reconnaître les apprentissages réalisés : le savoir appris (quoi et comment?).

5. Rappelons que si la Confédération produit des lois-cadres, ce sont les cantons qui fixent les modalités de mise en œuvre.

6. Le plan d'études romand (PER) s'applique à la Suisse romande puisqu'il a été harmonisée à cet échelon en secondaire 1 ; les approches sont très différentes dans les autres cantons suisses. Le paysage est absent de ce PER, il apparaît brièvement en $2^{\mathrm{e}}$ année du secondaire 2 dit gymnasial, 
où les programmes (très peu précis dans le système genevois) sont orientés vers une géographie plus « physique » ou « environnementale » selon les interprétations.

\section{RÉSUMÉS}

Le Grand Projet des Cherpines, le projet d'aménagement urbain de l'agglomération de Genève (Suisse) faisant débat, a été choisi comme cas d'étude commun dans le cadre d'un projet de recherche portant sur la didactique du paysage (2015-2018). Ce projet vise, à partir de cas analysés collectivement, à mutualiser les réflexions et propositions didactiques sur le paysage saisi comme outil de formation. Un workshop organisé en novembre 2015, a permis aux enseignants-chercheurs participants de confronter leurs regards et leurs méthodes et d'analyser une controverse selon une problématique décalée: analyser en quoi elle peut être un objet pertinent de formation et proposer des dispositifs pédagogiques pour des publics divers. Les résultats de cet exercice sont présentés brièvement au profit d'un bilan réflexif sur le potentiel didactique d'un tel cas d'étude, et sur les enjeux de formation au et par le paysage.

The controversial Cherpines major urban planning project in the agglomeration of Geneva (Switzerland) was chosen as a case study for a research project on landscape didactics (20015-2018). This project is intended, based on the collective analysis of cases, to share ideas and didactic propositions concerning the landscape as a training tool. A workshop organised in November 2015, enabled the research-professors taking part to compare their perceptions and methods and to address a controversial issue from a different angle: analysing how it can be a relevant training object and propose pedagogical approaches for different audiences. The results of this exercise are briefly presented in an evaluation of the didactic potential of such a case study and a reflection on the question of training in landscape architecture through the landscape.

\section{INDEX}

Mots-clés : didactique, paysage ordinaire, controverse, projet d'urbanisation, Grand Genève (Suisse)

Keywords : didactic, ordinary landscape, controversy, urban development project, Grand Genève (Switzerland)

\section{AUTEURS}

\section{ANNE SGARD}

Anne Sgard, géographe, est professeure associée au département de géographie et environnement de la faculté des sciences de la société et à l'Institut universitaire de formation des enseignants - université de Genève.

Anne.Sgard[at]unige[dot]ch 


\section{SYLVIE PARADIS}

Sylvie Paradis, géographe et architecte-urbaniste, est collaboratrice scientifique au département de géographie et environnement de la faculté des sciences de la société - université de Genève, et chercheure associée dans l'UMR Territoires de Clermont-Ferrand (issue du rapprochement des UMR Métafort et du Céramac).

sylvie.paradis[at]unige[dot]ch et sylvie.paradis[at]wanadoo[dot]fr 